\title{
Evaluating the Effect of Retinal Defocus (Blur) on Lateral Heterophoria
}

George Nnamdi Atuanya ${ }^{1}$, Osayimwen Osas Ero ${ }^{1}$, Raphael Uchenna Enyinnaya Akpalaba ${ }^{1}$

${ }^{1}$ Department of Optometry, Faculty of Life sciences, University of Benin, PMB 1154, Benin City, Edo state, Nigeria.

\begin{tabular}{l|l|l} 
Corresponding author: George Nnamdi Atuanya & Email: george.atuanya@uniben.edu & Phone: +2348060248631
\end{tabular}

\section{Abstract}

Purpose: This study was conducted to ascertain the effect of retinal defocusing or retinal blur on lateral heterophoria at far and near.

Methods: This was a quasi-experimental study involving 57 participants aged 5 to 35 years, who were selected based on the inclusion criteria of being myopes and emmetropes who had a normal general and ocular health. The Von Graefe technique was used for measuring phoria under standard testing conditions.

Results: The mean baseline lateral phoria for emmetropes were $0.53 \pm 1.14 \Delta$ exophoria and $2.47 \pm 2.08 \Delta$ exophoria at far and near respectively, while after defocusing, it was $2.80 \pm 0.88 \Delta$ exophoria and 4.34 $\pm 1.70 \Delta$ exophoria at far and near respectively. For myopes, the baseline lateral phoria was $1.52 \pm 1.09 \Delta$ exophoria and $4.76 \pm 1.13 \Delta$ exophoria at far and near respectively while after defocussing, it was $3.39 \pm$ $0.92 \Delta$ exophoria at far and $7.60 \pm 1.00 \Delta$ exophoria at near. The mean difference in lateral phoria was found to be significant at the $95 \%$ confidence level $(\mathrm{P}=0.000)$. There was no statistically significant difference in lateral phoria change at $\operatorname{far}(\mathrm{P}=0.072)$, while the near phoria difference was significant $(\mathrm{P}=0.002)$.

Conclusion: Gas Retinal defocusing with $+2.50 \mathrm{D}$ resulted in a shift towards exophoria at both far and near, with myopes experiencing more exophoric shift. This is because they are less sensitive to the presence of blur. This will enable practitioners to be cautious of the inhibition of accommodation due to higher exophoria at near when prescribing lenses.

\section{Keywords: Retinal Defocus, Myopia, Emmetropia, Near lateral phoria, Distance lateral phoria.}

\section{Introduction}

Heterophoria is the tendency for the visual axes of the two eyes not to be directed towards the point of fixation when fusion is interrupted ${ }^{1}$. Exophoria and esophoria is the turning of the eye outward and inward respectively from the straight-ahead position when fusion is broken1. Results on the distribution of distance heterophoria and near heterophoria have been well documented in literature ${ }^{2,3,4}$. There exist a high incidence of orthophoria at distance, although some researchers ${ }^{5,6}$ have reported

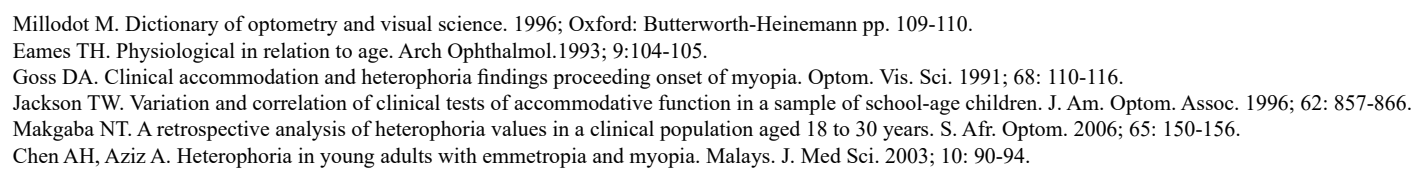


exophoria of about 0 to 6 prism diopters at near viewing distance. Heterophoria seems to vary with different viewing distances. Distance phoria was claimed to be different from tonic vergence due to accommodative divergence ${ }^{7}$. Emmetropia and myopia are the refractive states of the eye in which, with accommodation relaxed, the images of distant objects are focused on and in front of the retina respectively. Heterophoria has been reported to have close association with myopia development ${ }^{8}$. The near point esophoria has been shown to be associated with childhood myopia progression'. Late-onset myopes displayed higher mean AC/A and dark vergence values and lower mean dark-focus values than the subjects who remained emmetropic ${ }^{10}$. Retinal defocus is the ability of the eye to perceive a blur due to the addition of plus power lenses over the eyes. The reduction in visual resolution due to retinal blur is a common experience when individual requiring a corrective lens removes their spectacle or contact lenses ${ }^{11}$.

Heterophoria has been reported to have a close association with retinal defocus ${ }^{12}$, however, the role of refractive error (myopia) in its association should be determined. This therefore necessitated the need to investigate the effect of retinal defocus on lateral phoria at far and near in emmetropes and myopes. The aim of this study was to determine the effect of retinal defocusing or retinal blur on lateral heterophoria at far and near.

\section{Methods}

\section{Sample size determination and sampling technique}

The quasi experimental clinic-based study involving myopes and emmetropes of both sexes, was carried out at the University of Benin optometry clinics located in both campuses of Ugbowo and Ekenwan in Benin city, Edo State, Nigeria.

The sample size was calculated using the formula according to Huller et al., ${ }^{13}$.

$\mathrm{N}=\left[(1 / \mathrm{q} 1+1 / \mathrm{q} 2) \mathrm{E}(\mathrm{Z} \alpha+\mathrm{Z} \beta)^{2}\right]$

From the aforementioned formula, the minimum sample size was 55 based on these values: $(\mathrm{q} 1=0.5$, $\left.\mathrm{q} 2=0.5, \mathrm{Z} \alpha=1.96, \mathrm{Z} \beta=0.84, \mathrm{~S}^{2}=1, \mathrm{E}=0.75\right)$. The sample size was increased to 57 to take care of attrition.

A convenient sampling was used to select 25 myopes and 32 emmetropes to act as control for the study. Subjects included for the study were emmetropes and myopes who had a normal general and ocular health as well as those within the ages of 5 to 35 years of age. Those who were 36 years and above, hyperopes and subjects with ocular pathologies, as well as those on systemic medications which could affect phoria were excluded from the study.

\section{Methods of data collection:}

Emmetropia was defined as refractive power $\leq$ $+0.50 \mathrm{D}$ to $-0.50 \mathrm{D}$ in both eyes while myopia was referred to as refractive power greater than $-0.50 \mathrm{D}$ in both eyes. The instrument used for data collection include: a Phoropter, Trial lens set, Snellen VA charts, Retinoscope and Von Graefe targets for phoria far and near tests.

Refractive (objective and subjective) procedure was performed on the subject using standard procedures

\footnotetext{
Alfred DO, Richard AT. Lateral heterophoria at distance; Contribution of accommodation. Invest. Ophthalmol. Vis. Sci. 1992; 33: $2733-2743$.

Daniel A, Michel M. The possible effect of under-correction on myopic progression in children. J. Clinical and Experimental Optometry. 2006; 89: 315-321.

Rosenfield M, Hong SE, George S. Blur adaptation in myopes. Optom. Vis. Sci. 2004; 81: 657-662.

George S, Rosenfield M. Blur adaptation and myopia. Optom. Vis. Sci. 2004; 81: 543-547.

Rosenfield M, Abraham J, Cohen A. Blur sensitivity in myopes. Optom Vis. Sci. 1999; 76: 303-307.

Norton TT, Siegwart JT, Amedo AO. Effectiveness of hyperopic defocus, minimal defocus or myopic defocus in competition with a myopiagenic stimuli in tree shrew eyes. Invest.

Ophthalmol. Vis. Sci. 2006; 47(11): 4687-4699.

Huller V, Irvin D, Soo HR, Carolyn ZW. Sample size determination. Journal of Physics. 2013; 54: 157-166.
} 
and room illumination. Base line phoria test was performed using the Von Graefe technique at far $(6 \mathrm{~m})$ and near $(40 \mathrm{~cm})$, i.e. without the defocusing lens (+2.50D) and recorded. The phoria test was carried out with the defocusing lens (+2.50D) in place and the far and near phoria findings were recorded.

\section{The Von Graefe test Procedure}

A Von Graefe near phoria assessment was performed by asking the subject to fixate through the phoropter, with a near phoria target presented at $40 \mathrm{~cm}$. The subject was asked to appreciate the target before 12 prisms base- in were introduced into the right eye dial and six prisms base down into the left eye dial. The participants were asked to confirm seeing two sets of the near target, one slightly above and laterally displaced with respect to the other. The horizontal prism was then gradually reduced in unitary steps until the subject reported a vertical alignment of the two sets of targets. If the reported alignment with prism dial is in base-in direction, the participant is said to be exophoric up to the indicated amount of prisms left and vice versa. If the participant however reported alignment with the prism dial at zero, it was termed to be orthophoric ${ }^{14}$.

The baseline in phoria at far and near was then compared with the phoria after inducing the $+2.50 \mathrm{D}$ lens.

\section{Data Analysis}

Data was presented in tables and graphs. A comparison between lateral phoria between groups was done using the student $t$ - test, with the aid of the statistical package for social science (SPSS) version 22.0 software. The level of significance was set at $\mathrm{p}<0.05$.

Ethical consideration: Prior to the study, Ethical approval (With approval number EC/UBEN/LSC. OPT/18/034) was granted by the Department of Optometry, University of Benin Ethical Committee. Informed consent was sought from the subjects after explaining the procedures clearly to them, in accordance with the tenets of the Declaration of Helsinki for the use of human subjects.

\section{Results}

A total of a fifty seven (57) participants between the ages of 5 to 35 years with mean age of $23.75 \pm 4.23$ years participated in the study. It consisted of 29 males with a mean age of $23.97 \pm 4.18$ years and 28 females with a mean age of $23.54 \pm 4.34$ years. The $32(56 \%)$ emmetropes with a mean age of 24.31 \pm 2.99 were made up of 18 males and 14 females with mean ages of $24.94 \pm 2.78$ years and $23.50 \pm$ 3.13 years respectively while the 25 (44\%) myopes with mean age of $23.04 \pm 5.40$ years consisted of 11 males with mean age of $22.36 \pm 5.57$ years and 14 females with mean age of $23.57 \pm 5.42$ years. 
Table 1: Age description of population

\begin{tabular}{lccc} 
Refractive status & Mean Ages (SD) & & \\
\hline & Males & Females & Overall \\
Emmetropes & $24.94 \pm 2.78$ & $23.50 \pm 3.13$ & $24.31 \pm 2.99$ \\
Myopes & $22.36 \pm 5.57$ & $23.57 \pm 5.42$ & $23.04 \pm 5.40$ \\
Overall & $23.97 \pm 4.18$ & $23.54 \pm 4.34$ & $23.75 \pm 4.23$ \\
\hline
\end{tabular}

Table 2: Descriptive statistics of the baseline lateral phoria and phoria with the $+2.50 \mathrm{D}$ lenses at far $(6 \mathrm{~m})$ and near $(40 \mathrm{~cm})$.

\begin{tabular}{|c|c|c|c|c|c|}
\hline \multicolumn{2}{|c|}{ Refractive status } & $\begin{array}{l}\text { Baseline } \\
\text { phoria } @ \\
\text { 6m }\end{array}$ & $\begin{array}{l}\text { Baseline } \\
\text { phoria } @ \\
40 \mathrm{~cm}\end{array}$ & $\begin{array}{l}\text { Phoria with } \\
+2.50 D \text { lens } \\
\text { a } 6 \mathrm{~m}\end{array}$ & $\begin{array}{l}\text { Phoria with } \\
+2.50 D \text { lens } \\
\text { a } 40 \mathrm{~cm}\end{array}$ \\
\hline \multirow[t]{7}{*}{ Emmetropia } & $\mathrm{N}$ & 32 & 32 & 32 & 32 \\
\hline & Mean & 0.53 & 2.47 & 2.80 & 4.34 \\
\hline & Median & .00 & 3.00 & 3.00 & 4.50 \\
\hline & Mode & 0 & 3 & 2 & $4^{\mathrm{a}}$ \\
\hline & Std. Deviation & 1.14 & 2.08 & 0.88 & 1.70 \\
\hline & Minimum & -1 & -2 & 2 & 0 \\
\hline & Maximum & 3 & 5 & 5 & 8 \\
\hline \multirow[t]{7}{*}{ Myopia } & $\mathrm{N}$ & 25 & 25 & 25 & 25 \\
\hline & Mean & 1.52 & 4.76 & 3.39 & 7.60 \\
\hline & Median & 1.00 & 5.00 & 3.09 & 8.00 \\
\hline & Mode & 1 & 5 & 4 & 8 \\
\hline & Std. Deviation & 1.09 & 1.13 & 0.92 & 1.00 \\
\hline & Minimum & 0 & 3 & 2 & 6 \\
\hline & Maximum & 3 & 7 & 5 & 9 \\
\hline
\end{tabular}

a. Multiple modes exist. The smallest value is shown

The mean lateral phoria of emmetropes without the defocusing lenses was $0.53 \pm 1.14 \Delta$ exophoria and $2.47 \pm 2.08 \Delta$ exophoria at far and near respectively while with the lenses was $2.80 \pm 0.88 \Delta$ exophoria and $4.34 \pm 1.70 \Delta$ exophoria at far and near respectively. For myopes, the mean lateral phoria without the defocusing lenses was $1.52 \pm 1.09 \Delta$ exophoria and $4.76 \pm 1.13 \Delta$ exophorai at far and near respectively while with the lenses, $3.39 \pm 0.92 \Delta$ exophoria and $7.60 \pm 1.00 \Delta$ exophoria at far and near respectively was obtained. 
Table 3: Mean change in phoria at far $(6 \mathrm{~m})$ and near $(40 \mathrm{~cm})$

\begin{tabular}{llrrrr}
\hline & $\begin{array}{l}\text { Refractive } \\
\text { status }\end{array}$ & $\mathrm{N}$ & Mean & $\begin{array}{c}\text { Std. } \\
\text { Deviation }\end{array}$ & p-value \\
\hline the change in phoria & Emmetrope & 32 & 2.27 & 0.736 & \\
(dioptres) @ 6m & & & & & 0.072 \\
& Myope & 25 & 1.87 & 0.929 & \\
the change in phoria & Emmetrope & 32 & 1.88 & 1.157 & \\
(dioptres) @ 40cm & Myope & 25 & 2.84 & 1.106 & 0.002. \\
& & & & & \\
\hline
\end{tabular}

The mean change in phoria at far $(6 \mathrm{~m})$ after defocussing was $2.27 \pm 0.73$ and $1.87 \pm 0.93$ exophoria for emmetropes and myopes respectively, while the mean change in phoria at near $(40 \mathrm{~cm})$ was $1.88 \pm 1.16$ exophoria and $2.84 \pm 1.11$ exophoria for emmetropes and myopes respectively. A comparison of the change in lateral phoria of the two groups at far $(6 \mathrm{~m})$ showed no statistically significant difference (unpaired t-test, $p=0.072$ ) at $95 \%$ confidence level.

\section{Discussion}

The aim of this study was to determine the effect of retinal defocusing or retinal blur on lateral heterophoria at far and near.

The mean lateral phoria of emmetropes without the defocusing lenses was $0.53 \pm 1.14 \Delta$ exophoria and $2.47 \pm 2.08 \Delta$ exophoria at far and near respectively while in myopes, it was $1.52 \pm 1.09 \Delta$ exophoria and $4.76 \pm 1.13 \Delta$ exophoria at far and near respectively. These mean values can be compared with the (Optometric Extension Program (OEP) table of expected for phoria values ${ }^{14}$. It indicates that for individuals with normal binocular vision, lateral phoria values of $0.5 \Delta$ exophoria at $6 \mathrm{~m}$ to $6 \Delta$ at $40 \mathrm{~cm}$ resulted in a physiological exophoria of $5.5 \Delta$. Variation from the OEP table of expected values may be due to the sample size and also difference in the examiners.

With the defocussing lenses, the mean lateral phoria was $2.80 \pm 0.88 \Delta$ exophoria and $4.34 \pm$ $1.70 \Delta$ exophoria at far and near respectively in emmetropes. Consequently, the mean difference in lateral phoria of emmetropes at 6 meters and $40 \mathrm{~cm}$ was $2.27 \pm 0.74 \Delta$ exophoria and $1.88 \pm 1.16$ exophoria respectively. In the myopes, the mean lateral phoria with the lenses was $3.39 \pm 0.92 \Delta$ exophoria and $7.60 \pm 1.00 \Delta$ exophoria at far and near respectively indicating a mean difference of $1.87 \pm 0.93 \Delta$ and $2.84 \pm 1.11 \Delta$ in exophoria at 6 meters and $40 \mathrm{~cm}$ was respectively. These mean differences in both groups, were also found to be significant at the $95 \%$ confidence level $(\mathrm{P}=0.001)$. This is similar to a study ${ }^{15}$ where it was found that plus lens and prism combination decreased the lens induced exophoria. They also suggested that incorporating near base-in prisms when prescribing bifocal lenses for young progressive myopes with exophoria could reduce the positive lens- induced

15. Rosenfield M, Carrel MF. Effect of near addition lenses on the accuracy of the accommodative response. Optometry. 2001; 72: 19-24. 
oculomotor imbalance. There also was a shift towards exophoria although the change in exophoria was more at far than at near for emmetropes, unlike myopes where the induced exophoria was more at near. The reasons for these could be attributed to the components of convergence as well as the optical properties of plus lenses, which consist of series of base-in prisms stacked together base to base ${ }^{16}$. A prism is known to bend light towards its base while the image is directed towards the apex. For plus lenses, there would be displacement of the perceived image outwards. Therefore, the eye would have to move outwards to put the defocused image in focus, which could be termed an exophoric shift. A higher degree of exophoria may be expected at near due to the inhibition of accommodation ${ }^{17,18}$. At near, the $+2.50 \mathrm{D}$ defocusing lens is expected to reduce the amount of convergence or accommodative convergence (which is largely responsible for phoria at near) leading to divergence or exophoria as the stimulus to accommodation is reduced ${ }^{18,19}$. But accommodation still acts at this distance to control the divergence of the eyes and hence the exophoric shift. Some studies have implicated a cross-link between vergence and accommodation ${ }^{20}$. Therefore, as the stimulus to accommodation is reduced, convergence begins to drive accommodation resulting in a consequent decrease in the exophoria. This could have been the case in subjects at near. Therefore, adaptation to the $+2.50 \mathrm{D}$ lenses may occur at near ${ }^{21}$.

A comparison of the change in lateral phoria of the two groups at both far and near revealed no statistically significant difference at far (unpaired t-test, $p=0.072$ ) but not at near where the difference was significant (unpaired t-test, $p=0.002$ ). Kenneth et al., ${ }^{22}$ have showed that some aspects of accommodation may be slightly abnormal or different in myopes, compared with accommodation in emmetropes and hyperopes). A shift in vergence adaptation seen in myopes (compared to emmetropes) could be attributed to their higher accommodative adaptation ${ }^{23}$. It would be imperative and advised that more studies on blur adaptations be done with different degrees of ametropia on phoria and other visual functions.

\footnotetext{
Shapiro JA, Kelly JE, Howland HC. Accommodative state of young adult using reading spectacles. Vis. Res. 2005; 45:233-245.

Seidemann A, Schaeffel F. An evaluation of the lag of accommodation using photorefraction. Optom. Vis. Sci. 2003; 43: 419-430.

Cheng D, Schmid KL, Woo GC. The effect of positive lens addition and base-in prism on accommodation accuracy and near horizontal heterophoria in Chinese myopic children. J. Ophthalmic Physiol. Optics. 2008; 28: 225-237.

Ebenholtz SM, Fisher SK. Distance adaptation depends upon plasticity in the oculomotor control system. Perception and Psychophysics. 1982; 31: 551-560.

Rosenfield M, Gilmartin B. Accommodative adaptation induced by sustained disparity vergence. Am. J. Optom. Physiol. Optics. 1998; 65: 118-126.

Wang B, Ciuffreda KJ. Depth of focus of the human eye; Theory and clinical implications. Surv. Ophthalmol. 2006; 51:75-85.

Kenneth J, Ciuffreda KJ, Darren MW. Myopes show increased susceptibility to near-work after-effect. Invest. Ophthalmol. Vis. Sci. 1998: 39: 1797-1803.

Vidhyapriya S, Elizabeth LI, William RB. The effect of heterophoria type and myopia on accommodative and vergence responses during sustained near activity in children.

Vis. Res. 2012; 57: 9-17.
} 


\section{Conclusion}

This study has shown that retinal defocusing with $+2.50 \mathrm{D}$ resulted in a shift towards exophoria at both far and near due to the influence of accommodation. Myopes experience more exophoric shift as they are less sensitive to the presence of blur and therefore are less likely to experience a scenario where accommodation is activated by vergence to reduce exophoria. Clinicians should consider the impact of plus lenses on phoria in an attempt to determine if the progression of myopia can be impeded with plus lenses. It will also assist practitioners to be cautious when prescribing lenses due to its inhibitory effect on accommodation.

\section{Conflict of Interest}

There is no conflict of interest associated with this work. 